\title{
Proof-of-Principle Experiments for PHz Nano-Device using Semi- Metallization of Dielectrics under Strong Optical Fields
}

\author{
Dong Eon Kim \\ POSTECH / MPK
}

South Korea

\section{Extended Abstract}

Ultrafast charge transport and control by electromagnetic fields in semiconductors is an ever-growing demand for faster signal processing. Fascinating avenues for new phenomena and applications in solids has been opened by intense optical fields. The current switching and its control by an optical field may pave a way to PHz optoelectronic devices. Reversible semi-metallization in fused silica on a femtosecond time scale has been demonstrated by Schiffrin et. al. (Nature 493 70-74 (2013)). Wider spread of this technology demands better understanding of whether the strong field behavior is universally similar for different dielectrics. Here we demonstrate the universality of the physical picture explained by the localization of Wannier-Stark states. A carrier-envelope-phase stabilized, few-cycle strong optical field was employed to drive the semimetallization in sapphire, calcium fluoride and quartz. The comparison between these materials show its remarkable similarity in response of these materials, despite the significant differences in their physical properties. Our results pave the way toward PHz-rate optoelectronics. 